\section{Temperature fluctuations along food supply chain: A dynamic and stochastic predictive approach to establish the best temperature value in challenge tests for Listeria monocyto- genes}

\author{
Filippo Giarratana, ${ }^{1,2}$ Luca Nalbone, ${ }^{1,2}$ \\ Graziella Ziino, ${ }^{1,2}$ Giorgio Donato, ${ }^{2}$ \\ Stefania Maria Marotta, ${ }^{2}$ Filippa \\ Lamberta, ${ }^{2}$ Alessandro Giuffrida ${ }^{1,2}$ \\ ${ }^{1}$ Department of Veterinary Sciences, \\ University of Messina; ${ }^{2}$ RICONNEXIA \\ srls, Spin-off of the University of \\ Messina, Polo Universitario \\ dell'Annunziata, Messina, Italy
}

\section{Abstract}

This study aims to evaluate the behaviour of Listeria monocytogenes under fluctuating temperature comparing the efficacy of deterministic and stochastic methods for its prediction. In the first part of the study, a strain of L. monocytogenes was maintained at two different fluctuating temperature regimes both from 2 to $8^{\circ} \mathrm{C}$ and regularly sampled for the quantitative determination. The first temperature regime lasted 204 hours with a fluctuation length of 12 hours whereas the second lasted 167 hours with a fluctuation length of 24 hours. A dynamic predictive model was implemented for the reproduction of the observed data. Model resolution has been carried out by using values of the recorded temperature as well as the value of the mean temperature, the kinetic mean temperature, the $75^{\text {th }}$ and $95^{\text {th }}$ percentile of the temperature. A stochastic resolution was also performed considering the mean temperature and Standard Deviation as stochastic variable. In the second part of the study, a temperature mean curve was constructed by monitoring temperature of 8 refrigerated conveyances, 10 display cabinet and 15 domestic refrigerators. This curve was used to obtain predictive scenarios for L. monocytogenes based on the above and also considering temperature regime suggested by the EURL Lm TECHNICAL GUIDANCE DOCUMENT on challenge tests and durability studies for assessing shelf-life of ready-to-eat foods related to Listeria monocytogenes (Version 4 of 1 July 2021). All predicted behaviours were compared to the observed ones through the Root Mean Squared Error. Firstly, dynamic predictive model as well as the stochastic one, provided the best level of reproducibility of the observed data. The kinetic mean temperature reproduced the observed data better than the mean temperature for the 12 hoursregime while for the 24 hours-regime was the opposite. The $75^{\text {th }}$ and $95^{\text {th }}$ percentile overestimated the observed growths. Secondary, predictions obtained with the mean temperature, kinetic temperature and stochastic approach well fitted the observed data. The $75^{\text {th }}$ and $95^{\text {th }}$ percentile of Temperature and the "Eurl LM" temperature regimes overestimated the observed prediction. Dynamic approach as well as the stochastic one allowed to obtain the lowest values of Root Mean Squared Error. The mean temperature and kinetic mean temperature appeared the most representative values in a deterministic "single-point" approach.

\section{Introduction}

Nowadays, the food safety management approach is challenged by the global dimensions of food supply chains, the need for reduction of food waste and the environmental pollution that is the cause of the arising of emerging hazards (Uyttendaele et al., 2016; Nalbone et al., 2021). In the international context, borders are fading, especially for the globally traded commodities. There is a great divergence in the degree of organization, infrastructures, teaching capacity across countries and food safety can be compromised by specific hazards related to the country of origin (Arab et al., 2020,2021 ) as well as to the complexity of the food chain supply.

The cold chain for perishable products is significantly complex since it is articulated in several phases and on the responsibility of many stakeholders (Brenner, 2015). This complexity is, often, the cause of temperature abuses that affect the safety and quality of food and produce important economic losses. Some phases of the supply chain are well recognized as critical points such as the transport and the storage at the warehouse, retail, and domestic level. Derense et al. (2006) showed that temperature is critical in the last three phases of the cold chain: storage in display cabinet (7.3\%), transport after shopping (59.7\%) and domestic storage $(40.3 \%)$. These results were confirmed by Morelli and Derens (2009), Koutsoumanis et al. (2010) and Landfeld et al. (2011).

The results of the Frisbee European Project (Food Refrigeration Innovation, Safety Consumers' Benefit, Environmental Impact and Energy Optimisation, 2010-
Correspondence: Luca Nalbone, Department of Veterinary Science, University of Messina, Viale Palatucci snc- Polo Universitario Annunziata, 98168, Messina, Italy.

Tel: +390906766889 .

E-mail: lnalbone@unime.it

Key words: Food pathogens; Temperature challenge test; Storage condition; Domestic fridge; Display cabinet.

Conflict of interest: The authors declare no conflict of interest.

Contributions: The authors contributed equally.

Funding: None.

Availability of data and materials: All data and materials are available within the text.

Received for publication: 15 July 2021. Accepted for publication: 21 January 2022.

This work is licensed under a Creative Commons Attribution-NonCommercial 4.0 International License (CC BY-NC 4.0).

(C) Copyright: the Author(s), 2022

Licensee PAGEPress, Italy

Italian Journal of Food Safety 2022; 11:9981

doi:10.4081/ijfs.2022.9981

2014) confirm that the most sensitive links are transportation after purchase and, particularly, the storage in household refrigerators, while display cabinets temperature tends to be under better conditions than in the past (decrease in the mean product temperature and a lower percentage of products in which the temperature is higher than the specified temperature).

Concerning domestic storage, Roccato et al. (2017) carried out a comprehensive retrospective analysis of 15 European studies on household refrigerator temperature. Analysis of temperature distributions revealed two geographical groups: northern European countries and southern European countries. The overall variability of European domestic temperature refrigerators resulted described by a normal distribution: $7.0 \pm 2.7^{\circ} \mathrm{C}$ (mean standard \pm deviation) for southern countries, and $6.1 \pm 2.8^{\circ} \mathrm{C}$ for the northern countries. More recent research (Dumitrescu et al., 2020) about domestic refrigerator temperatures in five European countries (French, UK, Portugal, Romania and Norway), for specific consumer groups, has evidenced an overall average temperature of $6.3,4.6,5.5,5.7$ and 5.6 respectively; therefore, these results could be considered similar to those reported by Roccato et al. (2017). All the above surveys, besides confirming the cold chain weakness, raise 
two important matters. The former regards the choice of the best suitable approach for mimicking the temperature chain evolution in shelf-life studies since all cited data shows a wide temperature fluctuation at each supply chain level. The second concerns the use of a deterministic or a stochastic approach to predict microbial behaviour, since, according to some Authors (IAFP, 2013; Membré and Guillou, 2016; Roccato et al., 2017), the use of a single temperature value in mimicking the temperature chain ("single-point approach") would not consider the complexity of the environmental characteristics and the related influence on the bacterial population with a potential overestimation of the bacterial dynamics.

Concerning the shelf life studies, the EURL Lm TECHNICAL GUIDANCE DOCUMENT on challenge tests and durability studies for assessing shelf-life of ready-to-eat foods related to Listeria monocytogenes (Version 4 of 1 July 2021). individuate three time/temperature intervals in which divide the product shelf life: i) "From the manufacture until the arrival to the display cabinet": 1/3 of shelf life (or a maximum of 7 days if shelf life $>21$ days) with a temperature of $7{ }^{\circ} \mathrm{C}$ or the $95^{\text {th }}$ percentile of the Food Business Operator's data observation; ii) "Retail - display cabinet": $1 / 3$ of shelf life (or $1 / 2$ shelf life -7 days if shelf life is $>21$ days) with a temperature of $7^{\circ} \mathrm{C}$ or the $95^{\text {th }}$ percentile of the temperature observations for the country where the stage of the cold chain is located; iii) "Consumer storage": $1 / 3$ of shelf life (or $1 / 2$ shelf life 7 days if shelf life is $>21$ days) with a temperature of $12^{\circ} \mathrm{C}$ or the $95^{\text {th }}$ percentile of the temperature observations for the country where the stage of the cold chain is located. The use of the $95^{\text {th }}$ percentile of temperature has been considered by some authors (IAFP, 2013; Roccato et al., 2017) an unrealistic approach even for mimicking a "reasonable temperature abuse". A more realistic representation of temperature abuse along the food supply chain is probably given by the $75^{\text {th }}$ percentile of the temperature trend but, also in this case, it is about a "worst-case scenario" approach that does not consider the global effect of temperature fluctuation on microbial growth and, consequently, on shelf-life duration. Although all above-cited studies have analysed temperature trend along the food supply chain and have proposed a single point approach for its representation, only a few researchers have related the effect of temperature fluctuation on microbial growth and have identified a single temperature value that correctly reproduces the bacterial behaviour. The application of stochastic predictive models has been widely explored to reproduce the vari- ability of environmental parameters (eg. temperature fluctuation) and to assess the related microbial responses (Giuffrida et al., 2009; Koutsoumanis et al., 2010; Valenti et al., 2013, 2016). However, if the stochastic approach could reproduce the variability of microbial responses along with a risk assessment framework, it does not clarify the relationships among the reproduced stochastic scenarios and the individuation of single environmental values such as temperature, $\mathrm{pH}, \mathrm{aW}$, that can be used in shelf-life studies.

The present study aims to establish the relation between temperature fluctuations and the Listeria monocytogenes growth through a dynamic predictive approach, to assess the best "single point" method of representing the temperature trend, comparing a deterministic versus a stochastic modelling approach too.

\section{Materials and methods}

\section{Study planning}

The present study has been articulated in two main phases. In phase 1, cultures of a strain of Listeria monocytogenes (ATCC 13932), preincubated at $10^{\circ} \mathrm{C}$, were subjected to two kinds of experimental temperature regime; the former $(\operatorname{Tr} 12)$ fluctuated from $2 \pm 1^{\circ} \mathrm{C}$ to $8 \pm 1^{\circ} \mathrm{C}$ over a period of almost 12 hours and the latter ( $\operatorname{Tr} 24)$ over a period of almost 24 hours. Cultures were subjected to the temperature regimes "Tr12" and "Tr24" for 204 hours and 167 hours, respectively, and sampled at regular time intervals for the count of the L. monocytogenes. The temperature has been recorded with a data logger Smart-Vue ${ }^{\mathrm{TM}}$ 868MHz SV204-101-LSB (Thermo fisher Monza, Italy) set with a sampling frequency of 15 minutes.

Observed growth curves have been reproduced by a dynamic predictive model which considers, step by step, the recorded temperature fluctuations for both regimes (Tr12 and Tr24). The model (see below for details) has been used for further predictions with the mean temperature (mT), the mean kinetic temperature (mkT) (Giarratana et al., 2020), the $75^{\text {th }}$ and $95^{\text {th }}$ percentile of temperature. A further stochastic resolution of the predictive model has been carried out replacing each temperature recorded value with the Normal Distribution Value $(\mathrm{mT}$ value \pm standard deviation) of the temperature. In this case, the obtained growth prediction has been resulted by the mean value, for each step, of 1000 resolution (iterations) of the model. Iterations have been carried out by the Excel NORM.INV function (Microsoft
Corporation) that returns the inverse of the normal cumulative distribution for a specified mean and standard deviation value (in our case, the mean and standard deviation of observed temperature value). This function has been associated with the RANDOM function in order to obtain 1000 iterations. The agreement level between predicted and observed curves was measured by calculating the Root Mean Squared Error (RMSE).

Phase 2 of the study has aimed to acquire temperature data on the three main sectors of the food supply chain in order to obtain a mean trend of temperature for 21 days. Particularly, it has been monitored: i) the temperatures of transport from the manufacture until the arrival to the display cabinet; ii) the temperatures at retail in the display cabinet; iii) the household refrigerator temperatures. For the first sector, the temperature data of eight means of transports of cold-smoked salmon were considered; for the second part of the chain, the temperature of ten display cabinets of five large retailers have been monitored, considering both open and closed types; finally, concerning the domestic storage, fifteen household refrigerators have been monitored. Mean values for a time of seven days for each sector have been considered to construct a temperature trend of 21 days; this complete set of data has been used for the application of the aforementioned predictive dynamic model for L. monocytogenes and further predictions with mean temperature, mean kinetic temperature, 75th and 95th percentile of temperature and for the application of the dynamic stochastic model. A further kind of prediction, for this Phase of the study, has regarded the use of temperature profile suggested by the aforementioned "EURL Lm TECHNICAL GUIDANCE DOCUMENT for conducting shelf-life studies on Listeria monocytogenes in readyto-eat foods". The temperature values have been set at $7^{\circ} \mathrm{C}$ for 14 days and $12^{\circ} \mathrm{C}$ for 7 days (T Eurl-LM).

In order to evaluate the effectiveness of the predictions, the RMSE value has been calculated among the dynamic prediction that has been used as reference growth curve, in this case, and the other ones (mT, $\mathrm{mkT}, 75^{\text {th }}$ percentile, $95^{\text {th }}$ percentile, T EurlLM, stochastic prediction).

\section{Microbiological analysis}

According to the EURL Lm TECHNICAL GUIDANCE DOCUMENT (2019), one Cryobeads containing the strain of $L$. monocytogenes ATCC 13932 was transferred from the frozen stock $\left(-80^{\circ} \mathrm{C}\right)$ in $9 \mathrm{ml}$ of Tryptic Soy Broth (TSB) (Biolife, Milano, Italy) and incubated at $37^{\circ} \mathrm{C}$ for 
about 15-18 hours. After this period (time necessary for the organism to reach the early stationary phase about 9.20 Log $\mathrm{cfu} / \mathrm{ml}$ ) from this subculture (subculture 1), $100 \mu 1$ were transferred to $9 \mathrm{ml}$ of TSB and incubated a $10^{\circ} \mathrm{C}$ for 3 days in order to adapt the strain to the storage condition of phase 1 . The incubation period of 3 days is at $10^{\circ} \mathrm{C}$ the time necessary for the L. monocytogenes to reach the early stationary phase, to shorten the lag phase once inoculated.

Successive dilutions in $9 \mathrm{ml}$ tubes of TSB from the obtained subculture 2 were done until to reach the presumptive concentration of $4 \log _{10} \mathrm{cfu} / \mathrm{ml}$; from this last tube, instead, one $\mathrm{ml}$ was inoculated in six glass bottles with $99 \mathrm{ml}$ of TSB respectively, reaching the final required presumptive concentration of $2 \mathrm{Log} \mathrm{cfu} / \mathrm{ml}$. The so obtained six final L. monocytogenes suspensions were used for the two kinds of experimental temperature regimes, 3 for each condition. The results are reported as the mean value of these 3 repetitions.

The initial L. monocytogenes concentration and those of other intervals were enumerated on plates of Agar Listeria acc. to Ottaviani \& Agosti (ALOA $®)$ (Biolife, Milano, Italy) incubated at $37^{\circ} \mathrm{C}$ for $24-48$ hours as reported in the ISO 11290-2:2017 method.

\section{Predictive model}

The dynamic predictive model for $L$. monocytogenes has been implemented through the association of a primary and a secondary model. The primary model is the well-known Baranyi and Roberts (1994) model, represented by the following set of differential equations

$\frac{d N}{d t}=\mu_{\max } N \frac{Q}{1+Q}\left(1-\frac{N}{N_{\max }}\right)$

Eq. 1a $\frac{d Q}{d t}=\mu_{\max } Q$

where $N$ is the concentrations ( $\log \mathrm{CFU} / \mathrm{g}$ ) of L. monocytogenes at time $t ; \mu_{\max }$ is the maximum growth rates $\left(\mathrm{h}^{-1}\right)$ of $L$. monocytogenes; $N_{\max }$ is the maximum population densities ( $\log \mathrm{CFU} / \mathrm{g}$ ) of the microbial population; $Q$ represents the physiological state of the population (Baranyi and Roberts, 1994). In order to obtain a dynamical resolution of Eq. 1 (Giuffrida et al., 2009, 2013; Martinez-Rios et al., 2020), $\mu_{\max }$ was calculated at each variation in environmental parameters (here, every temperature value of each profile) using the secondary model proposed by Le Marc et al. (2002). Differential equations have been numerical(Microsoft Corporation) using the Euler method (Press et al., 2007). As above described, further resolutions have been obtained using a single temperature value (mean temperature - $\mathrm{mT}$; mean kinetic temperature $-\mathrm{mkT}$; 75 th and 95 th percentile of temperature; T Eurl-LM). The predictive model has been also resolved by introducing a stochastic variable for each Temperature value, represented by the normal distribution ( $\mathrm{mT} \pm$ standard deviation) of the temperature trend. In this case, the ly resolved into a spreadsheet of Excel

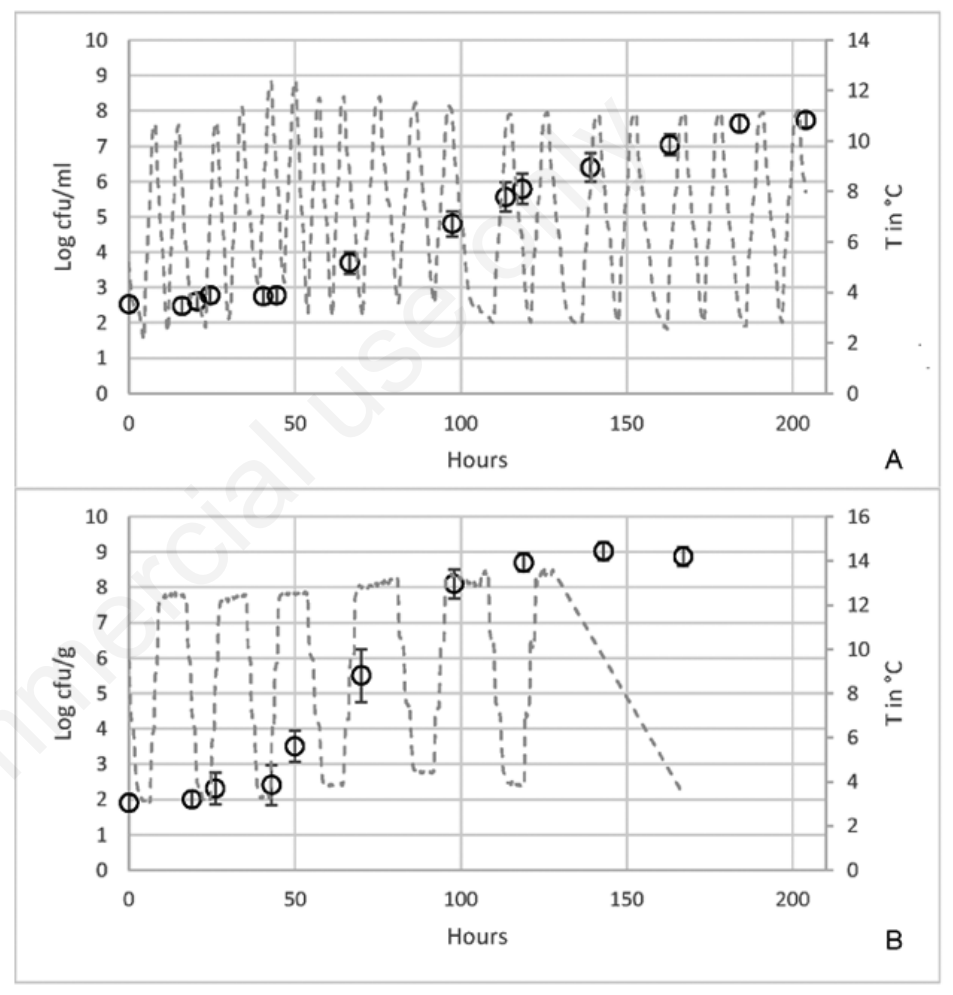

Figure 1. Temperature fluctuations (dotted lines) for $\operatorname{Tr} 12$ (panel A) and $\operatorname{Tr} 24$ (panel B) and related observed growth of $L$. monocytogenes (circles).

Table 1. Single value of representing the temperature profiles of phase 1 of the study.

\begin{tabular}{lcccc} 
Temperature regime & Mean temperature & Mean kinetic temperature & $7^{\text {th }}$ percentile temperature & $95^{\text {th }}$ percentile temperature \\
Tr12 & 6.95 & 7.45 & 9.30 & 11.27 \\
Tr24 & 8.97 & 9.91 & 12.53 & 13.33 \\
\hline
\end{tabular}

Table 2. RMSE values. For the phase 1, the RMSE value was calculated against the observed curves of each temperature profile; for the phase 2 , the dynamic prediction has been considered as curve of reference.

\begin{tabular}{|c|c|c|c|c|c|c|c|}
\hline & Dynamic & $\mathrm{mT}$ & mkT & $75^{\text {th }}$ perc. & $95^{\text {th }}$ perc. & Stochastic & Eurl-LM \\
\hline $\operatorname{Tr} 12$ & 0.1907 & 0.7271 & 0.2881 & 1.093 & 1.664 & 0.2423 & --- \\
\hline Tr24 & 0.2141 & 0.3372 & 0.5199 & 2.3312 & 2.6852 & 0.3271 & \\
\hline Phase 2 & --- & 0.1474 & --- & 0.7754 & 1.8608 & 0.0601 & 2.3152 \\
\hline
\end{tabular}


obtained curves resulted from the mean of the curves derived from 1000 iterations.

Concerning the predictions of Phase 1 of the study, $N_{0}$ and $N_{\max }$ have been set equal to the observed values for each mean curve of L. monocytogenes whereas the initial value of $\mathrm{Q}\left(Q_{0}\right)$ has been obtained by the fitting among the predicted curves and the observed ones, using the solver function of Excel (Microsoft Corporation). Furthermore, the application of the dynamic predictive model was carried out considering the $\mathrm{pH}$ and $\mathrm{aW}$ of the broth (7.00 and 0.997 respectively).

In Phase 2 of the research plan, the $Q_{0}$ has been set as the mean of $Q_{0}$ values of Phase 1 ( $Q_{0}$ for $\operatorname{Tr} 12$ and $Q_{0}$ of $\left.\operatorname{Tr} 24\right) ; N_{0}$ and $N_{\max }$ have been set equal to $\log 1.95$ $\mathrm{cfu} / \mathrm{g}$ and $\log 8.6 \mathrm{cfu} / \mathrm{g} ; \mathrm{pH}$ and AW were set at 6.2 and 0.980 respectively.

\section{Results}

Temperature trends and growths of

\section{Listeria monocytogenes}

The growth of L. monocytogenes at the temperature regimes $\operatorname{Tr} 12$ and $\operatorname{Tr} 24$ are shown in Figure 1. The growth at $\operatorname{Tr} 24$ resulted faster than the $\operatorname{Tr} 12$ one and reached the Nmax value before the $150^{\text {th }}$ hour than the curve at $\operatorname{Tr} 12$. This is easily explained because the wider period of the fluctuation in $\operatorname{Tr} 24$ has produced mean temperatures and mean kinetic temperatures higher than those of the $\operatorname{Tr} 12$, as shown in Table 1.

The predictive results against the observed ones are presented in Figure 2 for both $\operatorname{Tr} 12$ and $\operatorname{Tr} 24$. The dynamic predictions, as expectable, have always appeared in high agreement with the observed data as shown in Table 2 too, where are reported the RMSE values for each prediction. The mkT value produced a better prediction than the $\mathrm{mT}$ value for the $\operatorname{Tr} 12$ while, for the $\operatorname{Tr} 24$, $\mathrm{mT}$ produced a better agreement to the observed data, than the mkT. The mkT probably well expresses the overall effect of temperature fluctuations on microbial when the variations are very frequent as for the profile $\operatorname{Tr} 12$ whereas, for larger periods $(\mathrm{Tr}$ 24), the $\mathrm{mT}$ is enough for considering the temperature oscillations (Figure 2).

The predictions for the $75^{\text {th }}$ and $95^{\text {th }}$ percentile both of $\operatorname{Tr} 12$ and $\operatorname{Tr} 24$ have appeared widely overestimated for both profiles, in respect of observed data. The predictions obtained with the stochastic resolution of the model resulted in strict agreement with the observed data as confirmed by the RMSE values (Table 2) that are always closer to those obtained for the dynamic predictions. Therefore, in the case of experimental fluctuations ( $\operatorname{Tr} 12$ and $\operatorname{Tr} 24)$, the predictive stochastic model based on the normal distribution of the Temperature ( $\mathrm{mT}$ \pm standard deviation) appears a useful method of representing the relation between temperature fluctuations and bacterial behaviour, also showing the effectiveness of the mean temperature (or kinetic mean temperature) as more correct "single point" approach than the $75^{\text {th }}$ or $95^{\text {th }}$ percentiles.
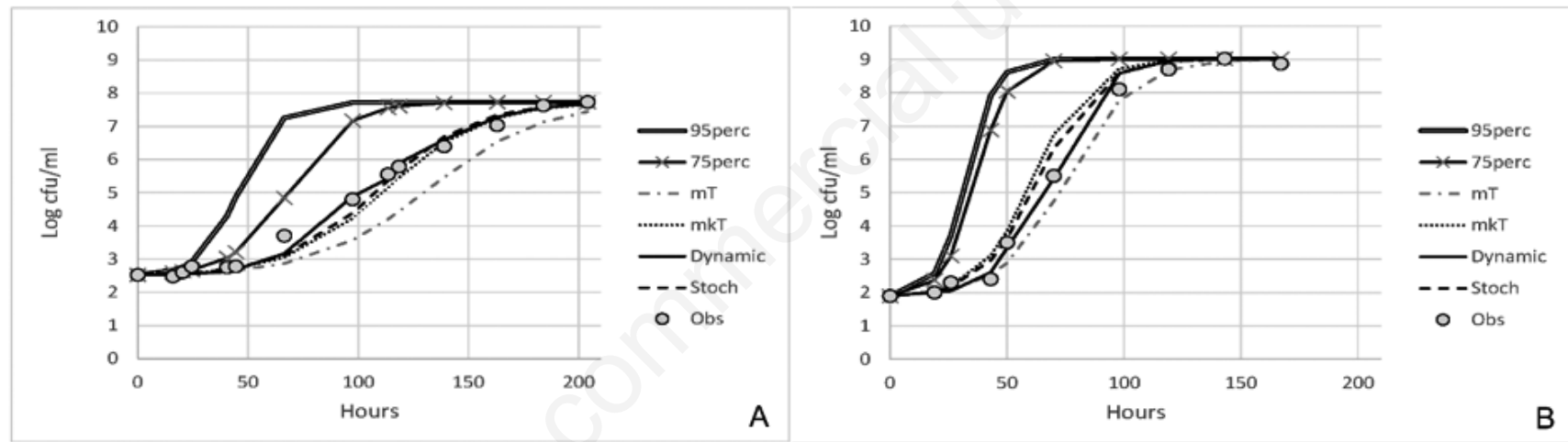

Figure 2. Observed (Obs) and predicted growth curves for $\operatorname{Tr} 12$ (panel A) and Tr24 (panel B).
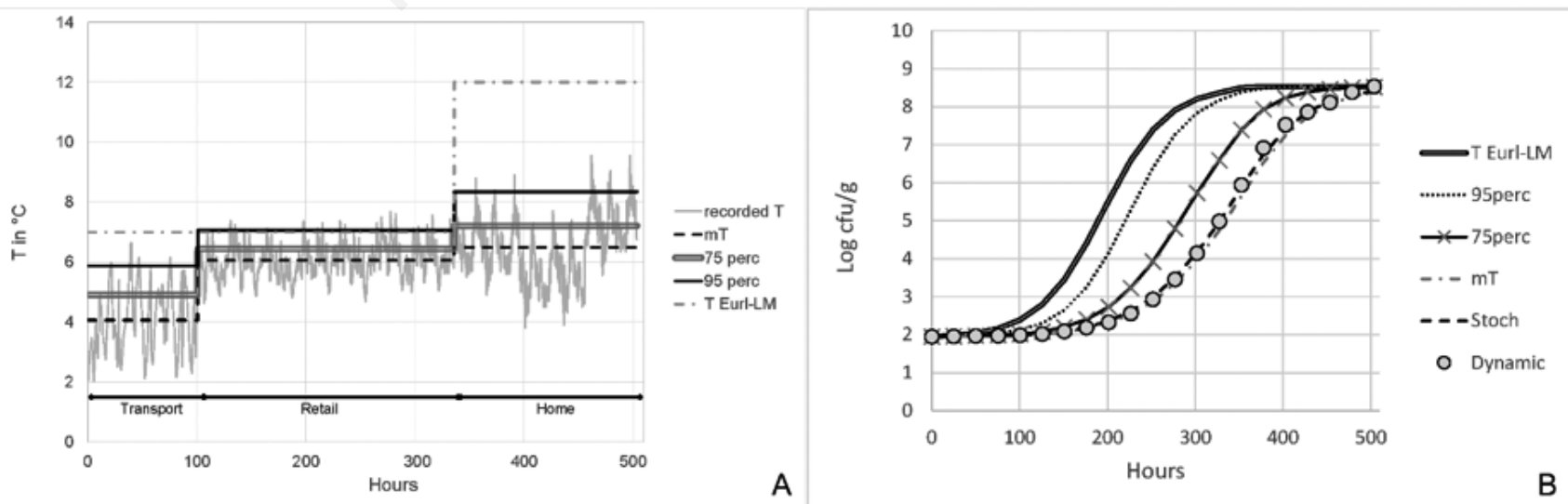

Figure 3. In panel A the temperature recorded for the entire food supply chain and related single values representing the temperature fluctuations for each sector ("Transport", "Retail", "Home"). In the panel B the dynamic prediction ("Dynamic") and further predicted growth curves for deterministic and stochastic representation of temperature fluctuations along the entire food supply chain. 


\section{Temperature along food supply chain and simulation of $L$. monocy- togenes behaviour}

The recorded mean trend temperature for the three sectors of the chain supply is reported in Figure 3 together with the mean temperature, the "T Eurl-LM", the 75th and 95th percentile of temperature, while the mean kinetic temperature for each sector has not been reported because it has resulted comparable to the mean one. Similarly, to phase 1 of the study (Table 1), also in this case there was a wide distance among mean temperatures and $75^{\text {th }}$ or $95^{\text {th }}$ percentile of temperature and this has been reflected on the related growth predictions. In this regard, as Figure 3 and Table 2 pointed out, the prediction with mean temperature has agreed to the dynamic one, which has been considered as a reference curve for this phase, according to the results of phase 1 . Conversely, the predictions with $75^{\text {th }}$ and $95^{\text {th }}$ percentile give an unrealistic scenario. Figure 3 shows also that the application of the temperature profile "T Eurl-LM" has produced the highest overestimation of $L$. monocytogenes growth.

Finally, also in this case, the application of the stochastic model has produced a prediction with the highest level of agreement to the curve of reference, giving a robust explanation on the meaning of mean temperature value as the most significant for the reproduction of the microbial behaviour under a fluctuating temperature regime.

\section{Discussion and conclusions}

In the last decade, the shelf life of refrigerated foods has been widely studied and debated from many points of view. Several studies have concerned the prolongation of perishable food shelf life using "natural compounds" (Trabelsi et al., 2019; Ed-Dra et al., 2020) and/or the application of the "hurdle technology" (Leistner, 2000; Singh and Shalini, 2016; Trabelsi et al., 2021). Many other studies have evaluated the applicability of shelf-life predictive models based on the characterization of the behaviour of pathogenic or spoilage microorganisms (Dalgaard, 1995; Neumeyer et al., 1997; Giuffrida et al., 2013; Martinez-Rios et al., 2016; Martinez Rios et al., 2020; Ed-Dra et al., 2021). In both cases, the knowledge of temperature history over the food chain supply has resulted in an extremely conditioning factor. For this reason, other research (Derense et al., 2006; Morelli and Derens, 2009; Landfeld et al., 2011; Roccato et al., 2017; Dumitrescu et al., 2020; ) have described the storage temperature evolution in the main sectors of the food chain stressing the thermal abuses and the related potential food safety impact. Regarding these data, the subsequent individuation of the $75^{\text {th }}$ or $95^{\text {th }}$ percentile of the temperatures has been the consequence of the need of representing these thermal abuses through a "single point" approach. This approach, however, contains a point of weakness because it does not consider the relation between temperature fluctuations and microbial behaviour, and it neglects the effect of low temperature values on the prolongation of the bacteria lag phase or the reduction of the growth rate. This effect is related to the frequency of the fluctuations as well as to other several parameters related to the food nature, and it cannot be neglected. On the other hand, the use of the $75^{\text {th }}$ percentile and, especially, of the $95^{\text {th }}$ percentile of the temperature cannot be simply considered a "worst-case scenario" approach because these values describe a partial region of the temperature fluctuations, neglecting the lower temperature bracket. Normally, as also observed in the present study, the temperature fluctuates equidistantly around the mean value, so a "worst-case scenario" should consider a high mean temperature but an equidistant fluctuation around the mean and not a fluctuation unbalanced towards high values. Therefore, the mean value of a temperature curve could be considered the best way to represent that curve with a single value since it considers the entire fluctuation around the mean value.

The results of the present study confirm the above considerations because the curves obtained with mean temperature values (or, in one case, with the mean kinetic temperature values) reproduce, with a good agreement $\left(\right.$ RMSE Tm $=0.1474$; RMSE $75^{\text {th }}$ perc. $=0.7754 ;$ RMSE 95 ${ }^{\text {th }}$ perc. $=1.8608$; RMSE EURL Lm = 2.3152), the observed curves and have appeared always very similar to the predictions obtained by the dynamic approach that considers each value of the temperature fluctuation but is applicable only if the entire temperature history is known. Also, the stochastic resolution of the predictive model has reproduced very well the observed (or reference) data, because it reproduces the influence of temperature fluctuations on the bacterial behaviour, better than the deterministic predictions. As well known (Giuffrida et al., 2009, Koutsoumanis et al. 2010, Koutsoumanis, 2016), stochastic predictive models are very useful in the risk assessment processes and, according to our results, this method can help to estimate the shelf life of a food category under a fluctuating temperature regime as normally occurs along with the food chain supply. When the deterministic approach must be applied (e.g., challenge or storage tests), the mean temperature value can produce a realistic scenario of storage since it includes the temperature abuses as well as the other fluctuation data.

The temperature mean values recorded in the present study for representing the entire food chain supply (phase 2), despite being referred only to a few distribution cycles, appeared very similar to those reported by the main above cited studies on the temperature of retail or domestic storage, therefore, the predictions obtained in phase 2 of the study confirm that the temperature profiles suggested by the "EURL Lm TECHNICAL GUIDANCE DOCUMENT for conducting shelf-life studies on Listeria monocytogenes in ready-to-eat foods" (here "T Eurl-LM" and 95"th percentile of Temperature") could produce unrealistic scenarios for the growth of $L$. monocytogenes in refrigerated ready to eat food and, consequently, for establishing the related shelf life.

\section{References}

Arab S, Nalbone L, Giarratana F, Berbar A, 2020. Occurrence of Vibrio spp. along the Algerian Mediterranean coast in wild and farmed Sparus aurata and Dicentrarchus labrax. Vet World 13:1199.

Arab S, Nalbone L, Giarratana F, Berbar A, 2021. Vibrio spp. in Wild and Farmed Mytilus galloprovincialis along the Algerian Mediterranean Coast: Evidence of V. cholerae 01 Serotype Ogawa. J Aquat Food Prod Technol 110.

Baranyi J, Roberts TA, 1994. A dynamic approach to predicting bacterial growth in food. Int J Food Microbiol 23:27794.

Brenner V, 2015. Causes of Supply Chain Disruptions. An Empirical Analysis in Cold Chains for Food and Pharmaceuticals. Springer Gabler, Wiesbaden, Germany.

Dalgaard P, 1995. Modelling of microbial activity and prediction of shelf life of packed fresh fish. Int J Food Microbiol 19:305-18.

Derens E, Palagos B, Guilpart J, 2006. The cold chain of chilled products under supervision in France. In: IUFOST, 13th World Congress of Food Science \& Technology "Food is Life", Nantes, France. https://iufost.edpsciences. org/articles/iufost/abs/2006/01/iufost06 000823 /iufost 06000823 .htm 1 . 
(Accessed 18 March 2020).

Dumitrescu L, Nicolau AI, Neagu C, Didier P, Maître I, Nguyen-The C, Skuland ES, Møretrø, T, Langsrud S, Truninger M, Teixeira P, Ferreira V, Martens L, Borda D, 2020. Time-temperature profiles and Listeria monocytogenes presence in refrigerators from households with vulnerable consumers. Food Control 111:107078

Ed-Dra A, Filali FR, Presti VL, Zekkori B, Nalbone L, Bouymajane A, Trabelsi N, Lamberta F, Bentayeb A, Giuffrida A, Giarratana F, 2020. Chemical composition, antioxidant capacity and antibacterial action of five Moroccan essential oils against Listeria monocytogenes and different serotypes of Salmonella enterica. Microb Pathog 149:104510.

Ed-Dra A, Nalbone L, Filali FR, Trabelsi N, El Majdoub YO, Bouchrif B, Giarratana F, Giuffrida A, 2021. Comprehensive Evaluation on the Use of Thymus vulgaris Essential Oil as Natural Additive against Different Serotypes of Salmonella enterica. Sustainability 13:4594

EURL Lm \& Ansens, 2019. Technical guidance document for conducting shelf-life studies on Listeria monocytogenes in ready-to-eat foods. Version 3 of 6 June 2014 - Amendment 1 of 21 February 2019. https://ec.europa.eu/ food/sites/ food/files/safety/docs/biosafety fh mc tech-guide-doc listeria-in-rtefoods_en.pdf. (Accessed 10 April 2020).

Frisbee European Project (Food Refrigeration Innovation, Safety Consumers' Benefit, Environmental Impact and Energy Optimisation, 20102014), 2014. Available from: https://cordis.europa.eu/project/id/2452 88/reporting/it. (Accessed 24 November 2020).

Giarratana F, Nalbone L, Ziino G, Giuffrida A, Panebianco F, 2020 Characterization of the temperature fluctuation effect on shelf life of an octopus semi-preserved product. Ital J Food Saf 9:48-53.

Giuffrida A, Valenti D, Ziino G, Spagnolo B, Panebianco A, 2009. A stochastic interspecific competition model to predict the behaviour of Listeria monocytogenes in the fermentation process of a traditional Sicilian salami. Eur Food Res Technol 228:767-75.

Giuffrida A, Valenti D, Giarratana F, Ziino G, Panebianco A, 2013. A new approach to modelling the shelf life of Gilthead seabream (Sparus aurata). Int J Food Sci Technol 48:1235-42.

IAFP's European Symposium on Food
Safety, 2013. How quantitatively "reasonably foreseen abuse" are included in shelf-life determination and risk assessment? Jeanne-Marie Membrè, INRA, France. http://www.researchgate. net/publications/313220210. (Accessed 10 April 2020).

ISO, 2017. Microbiology of the food chain - Horizontal method for the detection and enumeration of Listeria monocytogenes and of Listeria spp. - Part 2: Enumeration method. ISO Norm 11290-2:2017. International Organization for Standardization ed., Geneva, Switzerland.

Koutsoumanis K, Lianou A, Gougouli M, 2016. Latest developments in foodborne pathogens modelling. Curr Opin Food Sci 8:89-98.

Koutsoumanis K, Pavlis A, Nychas GJE, Xanthiakos K, 2010. Probabilistic model for Listeria monocytogenes growth during distribution, retail storage, and domestic storage of pasteurized milk. Appl Environ Microbiol 76:2181-91.

Landfeld A, Kazilova L, Houska M, 2011. Time temperature histories of perishable foods during shopping, transport and home refrigerated storage. In: International Conference of Refrigeration, Prague, Czech Republic. https://iifiir.org/fr/fridoc/27753. (Accessed 20 January 2020).

Le Marc Y, Huchet V, Bourgeois CM, Guyonnet JP, Mafart P, Thuault D, 2002. Modelling the growth kinetics of Listeria as a function of temperature, $\mathrm{pH}$ and organic acid concentration. Int $\mathrm{J}$ Food Microbiol 73:219-37.

Leistner L, 2000. Basic aspects of food preservation by hurdle technology. Int $\mathrm{J}$ Food Microbiol 55:181-6.

Martinez-Rios V, Bjerre Østergaard N, Gkogka E, Sand Rosshaug P, Dalgaard $\mathrm{P}, 2016$. Modelling and predicting growth of psychrotolerant pseudomonads in milk and cottage cheese. Int $\mathrm{J}$ Food Microbiol 216:110-20.

Martinez-Rios V, Gkogka E, Dalgaard P, 2020. Predicting growth of Listeria monocytogenes at dynamic conditions during manufacturing, ripening and storage of cheeses - Evaluation and application of models. Food Microbiol 92:103578.

Membré JM, Guillou S, 2016. Latest developments in foodborne pathogen risk assessment. Curr Opin Food Sci 8:1206.

Morelli E, Derens E, 2009. Evolution des temperatures du saumon fumé au cours des circuits logistiques. Revue générale $\mathrm{du}$ froid \& du conditionnement d'air
1090, 51-56. https://iifiir.org/fr/ fridoc/revue-generale-du-froid-ampamp-du-conditionnement-d-air-716. (Accessed 02 September 2010).

Nalbone L, Cincotta F, Giarratana F, Ziino G, Panebianco A, 2021. Microplastics in fresh and processed mussels sampled from fish shops and large retail chains in Italy. Food Control 125:108003.

Neumeyer K, Ross T, Mcmeekin TA, 1997. Development of a predictive model to describe the effects of temperature and water activity on the growth of spoilage pseudomonads. Int $\mathrm{J}$ Food Microbiol 38:45-54.

Press WH, Teukolsky SA, Vetterling WT, Flannery BP, 2007. Integration of Ordinary Differential Equations. In: Press WH, Teukolsky SA, Vetterling WT, Flannery BP, eds. Numerical Recepies: the Art of Scientific Computing. Cambridge University, New York, pp. 910-915.

Roccato A, Uyttendaele M, Membré JM, 2017. Analysis of domestic refrigerator temperatures and home storage time distributions for shelf-life studies and food safety risk assessment. Food Res Int 96:171-81.

Singh S, Shalini R, 2016. Effect of Hurdle Technology in Food Preservation: A Review. Crit Rev Food Sci Nutr 56:641-9.

Trabelsi N, Nalbone L, Di Rosa AR, Ed-Dra A, Nait-Mohamed S, Mhamdi R, Giuffrida A, Giarratana F, 2021. Marinated Anchovies (Engraulis encrasicolus) Prepared with Flavored Olive Oils (Chétoui cv.): Anisakicidal Effect, Microbiological, and Sensory Evaluation. Sustainability 13:5310.

Trabelsi N, Nalbone L, Marotta SM, Taamali A, Abaza L, Giarratana F, 2019. Effectiveness of five flavored Tunisian olive oils on Anisakis larvae type 1: application of cinnamon and rosemary oil in industrial anchovy marinating process. J Sci Food Agric 99:4808-15.

Uyttendaele M, Franz E, Schlüter O, 2016. Food Safety, a Global Challenge. Int J Environ Res Public Health 13:67.

Valenti D, Denaro G, Giarratana F, Giuffrida A, Mazzola S, Basilone G, Aronica S, Bonanno A, Spagnolo B, 2016. Modelling of Sensory Characteristics Based on the Growth of Food Spoilage Bacteria. Math Model Nat Pheno 11:119-36.

Valenti D, Giuffrida A, Ziino G, Giarratana F, Spagnolo B, Panebianco A, 2013. Modelling bacterial dynamics in food products: effects of environmental noise and interspecific competition. J Mod Phys 4:1059-65. 